\title{
Malignant Desmoplastic Type Mesothelioma Mimicking a Fibrous Pseudo- tumor of the Tunica Vaginalis: A Case Report and Literature Review
}

\author{
Fang-Yi Lee ${ }^{1}$, Mei-Chin Wen ${ }^{*}, 1,2$, John Wang ${ }^{1,2}$ and Chun-Kai Tseng ${ }^{3}$ \\ ${ }^{I}$ Department of Pathology, Taichung Veterans General Hospital, Taichung, Taiwan \\ ${ }^{2}$ College of Biomedicine and Nursing, HongKong University, Taichung, Taiwan \\ ${ }^{3}$ Department of Emergency Medicine, China Medical University Hospital, Taichung, Taiwan
}

\begin{abstract}
Malignant mesothelioma is an uncommon tumor of serosal surfaces. The most common sites of origin include the pleura and the peritoneum. Malignant mesothelioma of the tunica vaginalis testis is rare. We describe a 78 year-old man with a 2-month history of a rapidly enlarging scrotum. The initial pathologic diagnosis was fibrous pseudotumor by the presence of cellular spindle cells and the background of dense fibrous tissue. However, further immunohistochemistry revealed characteristic features of desmoplastic variant mesothelioma. Mesothelioma of the peritoneum and tunica vaginalis tends to be the epithelioid type. The purely desmoplastic-type mesothelioma of the tunica vaginalis has never been reported. Immunohistochemistry is essential to the diagnosis.
\end{abstract}

Keywords: Mesothelioma, desmoplastic mesothelioma, scrotal mass.

\section{INTRODUCTION}

Desmoplastic mesothelioma is an unusual subtype of malignant mesothelioma and constitutes approximately $10 \%$ of all malignant mesothelioma cases [1]. The disease generally arises from visceral pleura. The most common etiology of this neoplasm is asbestos exposure. Histologically, more than half of the tumor is fibrous and has low cell content [2]. Therefore, it can be difficult to distinguish a true neoplasm from a reactive process based on histology. In the present report, we describe a desmoplastic-type mesothelioma arising from the tunica vaginalis that was initially misdiagnosed as a fibrous pseudotumor.

\section{CASE REPORT}

A 78-year-old man presented 2-month history of rightsided scrotal swelling. He had suffered a straddle injury four years prior to this presentation and denied history of asbestos exposure. The results of other physical examination were unremarkable and the laboratory studies were within normal limits. Sonography showed right-sided multi-septum hydrocele. Then he underwent hydrocelectomy, and a white soft mass, measuring $3 \mathrm{~cm} \times 2 \mathrm{~cm} \times 1 \mathrm{~cm}$, was noted on the tunica vaginalis. The lesion was resected and submitted for pathologic examination.

The lesion was a solid and partly cystic tumor, which formed nodules on the surface of the tunica vaginalis. The intraoperative frozen section revealed that the tumor consisted of poorly defined fascicles and sheets of spindle cells, with oval nuclei, scant amphophilic cytoplasm and a few prominent nucleoli (Fig. 1A). The tumor cells displayed

*Address correspondence to this author at the Department of Pathology, Taichung Veterans General Hospital, No. 160, Sec. 3, Chung-Kang Rd., Taichung 40705, Taiwan; Tel: + 886-4-23592525, Ext. 5708; Fax: +886-423595046; E-mail: rosehairlee@gmail.com abundant intercellular collagen deposition and a haphazard arrangement of slit-like spaces (Fig. 1B). The initial pathologic diagnosis was nodular fibrous proliferation (fibrous pseudotumor) of the tunica vaginalis testis. Further immunohistochemical studies were performed. The tumor cells were immunoreactive for cytokeratin (Fig. 1C) and calretinin (Fig. 1D), and negative for CD34, CEA, desmine and S-100 protein. Based on these findings, the final diagnosis was desmoplastic-type mesothelioma. The patient underwent radical orchiectomy. At 8-month postoperative follow up, abdominal and pelvic computed tomography (CT) revealed no tumor recurrence.

\section{DISCUSSION}

Malignant mesothelioma of the tunica vaginalis is a rare, aggressive tumor with a wide age range of occurrence and a wide morphological spectrum [3]. Patients typically present with hydrocele, but initial physical examination rarely suggests malignancy [3]. A history of asbestos exposure and trauma has been proposed as an etiological factor for this tumor $[1,3]$. Malignant mesothelioma can be subclassified into epithelioid, sarcomatoid, desmoplastic and biphasic variants depending on the dominant histologic type. Each type shows nuclear pleomorphism, mitotic activity and stromal invasion. Immunohistochemistry is helpful in reaching the correct diagnosis $[4,5]$.

The initial intraoperative frozen biopsy specimen from our patient revealed a thickened tunica vaginalis consisting of densely fibrous paucicellular tissue. The fibroblast-like spindle cells were bland-looking and had low cell content. At first, this was attributed to inflammatory involvement of the surrounding tissue; however, it was later determined that the spindle cells infiltrated the adjacent soft tissue. They were also immunohistochemically positive for cytokeratin and the mesothelial marker calretinin. Based on these findings, desmoplastic-type mesothelioma was diagnosed. In our case, 


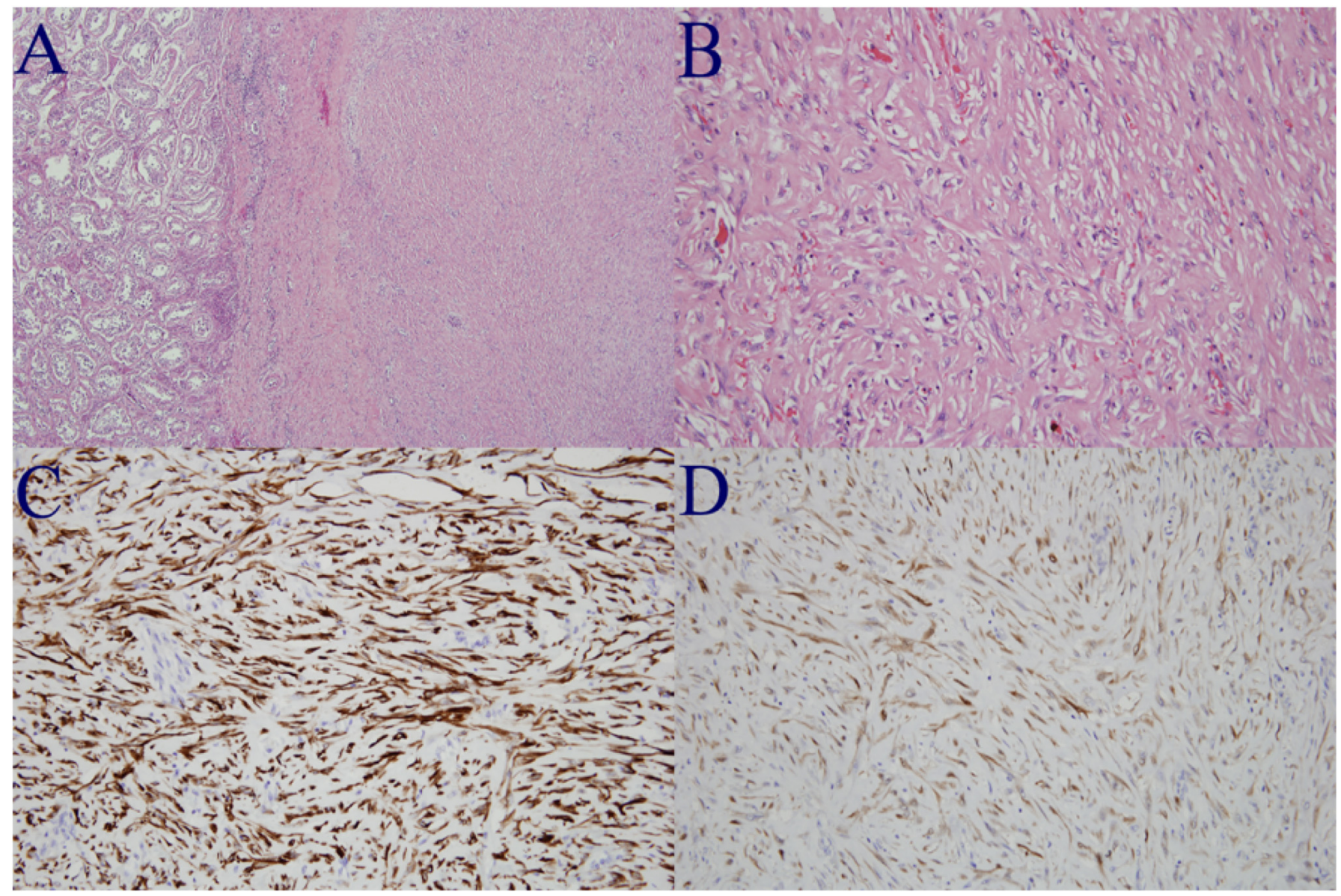

Fig. (1). The right side area showed dense collagenized tissue infiltrated by atypical spindle cells arranged in patternless pattern adjacent to right side seminiferous tubules (A). Haphazard arrangements of the slit-like spaces were noted of tumor (B). The neoplastic spindle cells were immunoreactive for cytokeratin $(\mathbf{C})$ and the mesothelial marker calretinin (D).

fibrous paucicellular tissue comprised more than $90 \%$ of the thickened tunica vaginalis, and the histological features of fibroblast-like cells were indicative of the desmoplastic variant.

The differential diagnosis of desmoplastic mesothelioma of the tunica vaginalis includes fibrous pseudotumor, solitary fibrous tumor, primary sarcoma, non-specific reactive fibrosis and reactive mesothelial hyperplasia. The tumor cells in our case were immunoreactive for cytokeratin and negative for CD34, which helped us differentiate it from a solitary fibrous tumor. Although the desmoplastic areas in fibrous pseudotumors of the tunica vaginalis resemble those seen in desmoplastic mesothelioma, the presence of keratin and calretinin indicate the desmoplastic mesothelioma could be considered more than fibrous pseudotumor. Primary sarcoma of tunica vaginalis is rare and often cytokeratin negative. In inflammatory and reactive lesions, there is usually granulation tissue with inflammatory cell infiltration. The fibrous tissue of inflammation does not show a basket-weave pattern with haphazard arrangement of slit-like spaces. In addition, the cytokeratin-positive atypical spindle cell infiltration of peripheral adipose tissue is not a feature of reactive fibrous lesions. Distinguishing malignant mesothelioma from benign fibrous lesions of the tunica vaginalis is clinically important. Orchiectomy may be unnecessary when the neoplasm is benign. Intraoperative frozen section analysis is helpful in es- tablishing the correct diagnosis and is essential for testicularsparing procedures. In malignant mesothelioma, although adjuvant chemotherapy and radiotherapy have little value, local and nodal recurrence may be successfully treated by orchiectomy with en bloc excision of the scar and underlying tissue [6].

With reference to the etiology of desmopastic mesothelioma of the pleura, $75 \%$ of patients had previous exposure to asbestos, but cases without asbestos exposure have also been reported [1]. The etiology of tumorigenesis of mesothelioma of the tunica vaginalis remains unknown. It has been suggested that some malignant mesothelioma might be due to local trauma, inflammation and asbestos exposure [3, 7, 8]. In our case, the patient denied a history of asbestos exposure but he had suffered a straddle injury four years prior to this presentation. In addition to asbestos exposure, infection by SV40 virus has recently been shown to be an etiological factor for malignant mesothelioma of pleura. However, SV40 DNA has not been investigated in peritoneal or tunica vaginalis mesothelioma [1]. To facilitate the understanding of the etiology of malignant mesothelioma of peritoneum and tunica vaginalis, a systemic study involving many cases is needed.

In summary, this is the first case of desmoplastic-type mesothelioma of the tunica vaginalis. This is a rare variant of malignant mesothelioma and is often misdiagnosed as reac- 
tive fibrosis because of extensive collagen fibrosis. For further treatment, it is important to recognize this variant and to distinguish it from non-specific fibrosis, solitary fibrous tumor and sarcoma of the tunica vaginalis. Malignant mesothelioma must be considered in patients with a clinical diagnosis of testicular hydrocele. Although malignant mesothelioma of the tunica vaginalis is seldom suspected or confirmed preoperatively, a biopsy specimen should be taken when the diagnosis is suspected. The history of asbestos exposure and trauma, and a number of histological criteria, such as foci of bland collagen necrosis, invasion of adipose tissue, as well as reliable immunohistochemical studies are necessary to make the correct diagnosis.

\section{REFERENCES}

[1] Ishikawa R, Kikuchi E, Jin M, et al. Desmoplastic malignant mesothelioma of the pleura: Autopsy reveals asbestos exposure. Pathol Int 2003; 53: 401-6.
[2] Wilson GE, Hasleton PS, Chatterjee AK. Desmoplastic malignant mesothelioma. A review of 17 cases. J Clin Pathol 1992; 45: 295-8.

[3] Jones MA, Young RH, Scully RE. Malignant mesothelioma of the tunica vaginalis. A clinicopathologic analysis of 11 cases with review of the literature. Am J Surg Pathol 1995; 19: 815-25.

[4] Plas E, Riedl CR, Pfluger H. Malignant mesothelioma of the tunica vaginalis testis: Review of the literature and assessment of prognostic parameters. Cancer 1998; 83: 2437-46.

[5] King JE, Hasleton PS. Immunohistochemistry and the diagnosis of malignant mesothelioma. Histopathology 2001; 38: 471-76.

[6] Eden CG, Bettochi C, Coker CB, Yates-Bell AJ, Pryor JP. Malignant mesothelioma of the tunica vaginalis. J Urol 1995; 153: 10534.

[7] Fligiel Z, Kaneko M. Malignant mesothelioma of the tunica vaginalis propria testis in a patient with asbestos exposure. A case report. Cancer 1976; 37: 1478-84.

[8] Karunaharan T. Malignant mesothelioma of the tunica vaginalis in an asbestos worker. J R Coll Surg Edinb 1986; 31: 253-54.

Received: December 9, 2008

Revised: December 17, 2008

Accepted: December 24, 2008

(C) Lee et al.; Licensee Bentham Open.

This is an open access article licensed under the terms of the Creative Commons Attribution Non-Commercial License (http://creativecommons.org/licenses/by$\mathrm{nc} / 3.0 /$ /) which permits unrestricted, non-commercial use, distribution and reproduction in any medium, provided the work is properly cited. 